\title{
VULNERABILIDADE SOCIOECONÔMICA DA CIDADE DE FORTALEZA AO COVID-19: O EPICENTRO DA PANDEMIA NA REGIÃO NORDESTE DO BRASIL
}

\author{
Samuel Façanha Câmara \\ Universidade Estadual do Ceará, Fortaleza, CE, Brasil \\ sfcamara2000@gmail.com \\ Hermano José Batista de Carvalho \\ Universidade Estadual do Ceará, Fortaleza, CE, Brasil \\ hermano.carvalho@uece.br \\ Felipe Gerhard \\ Universidade Estadual do Ceará, Fortaleza, CE, Brasil \\ gerhard.sousa@aluno.uece.br \\ Felipe Roberto da Silva \\ Universidade Estadual do Ceará, Fortaleza, CE, Brasil \\ felipe.roberto@aluno.uece.br \\ Thiago Matheus de Paula \\ Universidade Estadual do Ceará, Fortaleza, CE, Brasil \\ thiago.paula@aluno.uece.br
}

\begin{abstract}
RESUMO
Esta pesquisa objetivou identificar a vulnerabilidade associada à saúde e às dimensões social e econômica dos moradores dos bairros de Fortaleza quanto à pandemia Covid-19. Em seus aspectos metodológicos, o estudo exploratório-descritivo de natureza quantitativa mensurou a vulnerabilidade dos bairros da cidade de Fortaleza em três etapas: i. Vulnerabilidade Ampla - constituída por vínculos empregatícios, renda anual e pobreza extrema presente em cada bairro; ii. Vulnerabilidade Territorial - formada por aspectos relacionados às esferas econômica e sociodemográfica; iii. Vulnerabilidade das condições de Saúde - constituída, além das vulnerabilidades anteriores, por fatores como oferta de serviços de saúde. Os resultados do mapeamento ressaltaram a divisão econômica e social presente em Fortaleza. Enquanto os bairros mais ricos se destacam na análise por apresentarem uma alta vulnerabilidade econômica, ligada à produção, os mais pobres se destacam pela alta vulnerabilidade social. Essa discrepância suscita a possibilidade de existência de duas crises pandêmicas distintas exigindo a formulação de estratégias completamente diferentes para impedir os seus avanços. Destaca-se, ademais, uma preocupação adicional relacionada ao combate da Covid-19 nas Zonas Especiais de Interesse Social, contextos sociais marcados pela pobreza e precariedade da estrutura pública.
\end{abstract}

Palavras-chave: Vulnerabilidade socioeconômica. Surto pandêmico. Coronavírus. Bairros. Zonas Especiais de Interesse Social.

\section{SOCIOECONOMIC VULNERABILITY OF THE FORTALEZA CITY TO COVID- 19: THE PANDEMIC EPICENTER IN NORTHEAST BRAZIL}

\begin{abstract}
This research aims to identify the vulnerability associated with health and the social and economic dimensions of the residents of the neighborhoods of Fortaleza, capital and epicenter of the state of Ceará regarding the Covid-19 pandemic. As methodological aspects, an exploratory-descriptive study with quantitative nature measured the vulnerability of neighborhoods in the city of Fortaleza in three stages: i. Wide Vulnerability - consisting of employment bonds, annual income and extreme poverty present in each neighborhood; ii. Territorial Vulnerability - formed by aspects related to the economic and sociodemographic spheres; iii. Vulnerability of Health Conditions - constituted, in addition to previous vulnerabilities, by factors such as offering health services. The results of the mapping highlighted the economic and social division present in Fortaleza. While the wealthiest neighborhoods stand out in the analysis for presenting a high economic vulnerability, linked to production, the poorest ones stand out for their high social vulnerability. This discrepancy raises the possibility of two distinct pandemic crises requiring the formulation of completely different strategies to prevent their progress. In addition, there is a further concern related to the fight against Covid-19 in Special Zones of Social Interest, social contexts marked by poverty and precarious public structure.
\end{abstract}

Keywords: Socioeconomic Vulnerability. Pandemic Outbreak. Coronavirus. Neighborhoods. Special Zones of Social Interest. 
Samuel Façanha Câmara

Hermano José Batista de Carvalho

Felipe Gerhard

Vulnerabilidade socioeconômica da cidade de Fortaleza ao

Felipe Roberto da Silva

Covid-19: o epicentro da pandemia na região Nordeste do Brasil

Thiago Matheus de Paula

\section{INTRODUÇÃO}

Existe, atualmente, uma complexidade na dinâmica espaço-temporal das pandemias no mundo globalizado, uma vez que estas dependem de diversas variáveis como, interação, fluxo humano, densidade demográfica, assim como a composição dos centros populacionais (MCLAFFERTY, 2010; KRAEMER et al., 2020). Problemas de saúde coletiva, resultados desses surtos patológicos, entram no escopo de fenômenos perversos, já que estes problemas carregam algumas singularidades, permitindo múltiplas e controversas soluções (WALTNER-TOEWS, 2017; XIANG, 2013). Rittel e Webber (1973) explicam que estes fenômenos são caracterizados por apresentarem esforços mobilizados para solucioná-los e ocorrerem a partir de informações pouco precisas e, muitas vezes, por meio da tentativa e erro, uma vez que não há referências anteriores que permitam adotar um modelo de governança pré-estabelecido para momentos de crise.

Percebe-se, então, que a expansão geográfica de um surto pandêmico é resultado da rápida propagação dos fluxos globais de pessoas pelas redes de transporte, como é o caso da pandemia de COVID-19, causada pelo novo coronavírus, a qual vem gerando sérias consequências não só para a saúde coletiva, mas também para a economia, meio ambiente e sociedade (MCLAFFERTY, 2010). Este surto se desencadeou na cidade de Wuhan, localizado na província de Hubei, na China, e já se espalhou por todos os continentes. Até o último balanço de 18 de agosto de 2020, 21,9 milhões de pessoas foram contaminadas em todo mundo, concentrando a maioria dos casos nos Estados Unidos, Brasil e Índia, os quais acumulam 11,5 milhões de infectados (>52,5\%) (JHU, 2020).

Só no Brasil, conforme os dados do Ministério da Saúde (MS, 2020), neste mesmo período, foram registrados 3,3 milhões de casos confirmados de Covid-19, resultando em 108 mil mortes, o que preocupa as autoridades governamentais e a sociedade no geral, uma vez que, além da alta transmissibilidade do vírus, existe um contexto político, social e econômico que deve ser levado em conta. Mclafferty (2010) salienta que as comunidades são afetadas por patógenos de forma desigual, dado que a suscetibilidade irá depender de suas habilidades para lidar com as doenças e resistir a elas, o ambiente no qual está inserido, assim como seu acesso a tratamentos eficazes. Dessa forma, percebe-se que a vulnerabilidade desses grupos e comunidades pode variar em diferentes escalas, parametrizando sua posição social, econômica, bem como seu estado físico e biológico.

Uma gama de estudos tem analisado a vulnerabilidade sob diferentes perspectivas. Por exemplo, as dimensões ecológica e ambiental têm sido bastante exploradas (e.g. HE; SHEN; ZHANG, 2018; LINSDE-BARROS, 2017; NGUYEN et al., 2016; DE LANGE et al., 2010), assim como as físicas e espaciais (e.g. NELSON; GRUBESIC, 2018; SINGLETON et al., 2016). As dimensões social e econômica foram estudadas de forma isolada, ou associada aos aspectos de saúde (e.g. HOM et al., 2018; SILVA; KAWASAKI, 2018; ADHIKARI et al., 2016; PAVEGLIO et al., 2016). Porém, pouquíssimas pesquisas começam a aparecer tendo como lente a vulnerabilidade associada à saúde e às dimensões social $e$ econômica, dentro do escopo de fenômenos perversos específicos, como a atual pandemia causada pela COVID-19.

Ressalta-se a importância deste estudo, uma vez que a cidade de Fortaleza (epicentro da região Nordeste), tem acumulado, até o levantamento de 18 de agosto de 2020, 45.063 casos confirmados (SESA, 2020). Além disso, salienta-se o atual quadro de desigualdade social do município, visto que, por meio da análise comparativa do padrão de vida das comunidades de moradores, existe uma grande disparidade no Índice de Desenvolvimento Humano (IDH) entre os bairros. Cabe informar que o bairro Meireles tem o maior IDH da cidade (0,9530), quase oito vezes maior que o IDH do Conjunto Palmeiras $(0,119)$ (PMF, 2014). Assim, a opulência e a miséria na cidade desnivelam o grau de exposição e suscetibilidade dos indivíduos a essa ameaça, principalmente em territórios de baixa renda e em condições de pobreza, como são as Zonas Especiais de Interesse Social (ZEIS).

Destarte, surge o seguinte questionamento: Qual a vulnerabilidade associada à saúde e às condições social e econômica dos moradores dos bairros do município de Fortaleza? Logo, estabeleceu-se como 
Samuel Façanha Câmara

objetivo da pesquisa identificar a vulnerabilidade associada à saúde e às condições social e econômica dos moradores dos bairros de Fortaleza quanto à pandemia de Covid-19.

\section{VULNERABILIDADE SOCIAL E POBREZA URBANA}

Em um contexto geral, a vulnerabilidade é definida na literatura como um construto multidimensional, o qual avalia a relação entre o grau de exposição e suscetibilidade de pessoas, sistemas, comunidades ou sociedades a tensões específicas e suas respectivas capacidades para lidar com elas (NELSON; GRUBESIC, 2018; MCLAFFERTY, 2010). Atuando em diferentes espaços sociais, o enfoque da vulnerabilidade transita, portanto, em dois principais tipos de conceitualizações: físicas e coletivas (WU; YARNAL; FISHER, 2002). A primeira perspectiva estima a vulnerabilidade como potencial exposição a um risco físico ou perigo, cujo enfoque é voltado às dimensões ecológicas e ambientais de ameaças dos desastres naturais e mudanças climáticas (e.g. LINS-DE-BARROS, 2017; TIBÚRCIO; CORRÊA, 2012; CUTTER et al., 2008). A segunda conceitualização, por sua vez, avalia a reposta social à vulnerabilidade, desde suas capacidades políticas, econômicas e institucionais de reação à ameaça (WU; YARNAL; FISHER, 2002).

A partir de sua perspectiva social e econômica, entende-se por vulnerabilidade a exposição a determinada ameaça e seus efeitos de magnitude material ou moral sobre a população (HAND et al., 2018; EMRICH; CUTTER, 2011; PATNAIK; NARAYANAN, 2009). Dentro desse panorama, ameaças pandêmicas como o Covid-19 podem ser compreendidas como choques externos que alteram a dinâmica espacial, gerando inseguranças política, biológica, econômica e social (LI et al., 2020; JIA et al., 2020). Apesar disso, a exposição de pessoas a ameaças externas ocorre de modo desigual, variando conforme a disposição do espaço social e das privações individuais (MCLAFFERTY, 2010).

O processo de exposição às doenças infecciosas está intimamente relacionado à desigualdade social, à pobreza e à marginalização social (NEIDERUD, 2015; MCLAFFERTY, 2010). Características da pobreza urbana podem atuar como foco da transmissão de doenças e acelerar a propagação de patógenos em virtude de sua constituição espacial marcada pela alta densidade populacional em aglomerados subnormais ou em favelas, representados, em grande medida, por assentamentos informais (BAKER, 2012; LALL; DEICHMANN, 2010). Ademais, circunstâncias da pobreza, como a insegurança alimentar, podem enfraquecer o sistema imunológico, enquanto sua dimensão social de limitado acesso a serviços de saneamento e saúde adequado recrudescem as taxas de transmissão e aumentam a morbimortalidade (NEIDERUD, 2015; MADHAV et al., 2017).

Em conjunto, esses fatores sugerem que grupos sociais marginalizados e vulneráveis à pobreza, que vivem em condições socioespaciais de aglomerações subnormais com ausência de serviços essenciais básicos, são, provavelmente, os grupos que enfrentam maiores riscos de morbimortalidade durante uma pandemia. Além da elevada exposição, ambientes de exclusão social reduzem a capacidade de resiliência dos pobres urbanos a esse tipo de pandemia, ao passo que seu processo de contágio é reforçado pela pobreza, o que pode aprofundar ainda mais o empobrecimento das famílias (BAKER, 2012; LALL; DEICHMANN, 2010).

Contextos de epidemias limitam a capacidade de retorno da vida produtiva após a infecção, sobretudo devido ao escasso suporte social e econômico ofertado por instituições governamentais (MCLAFFERTY, 2010). De fato, a International Labour Organization (ILO, 2020) estima que 25 milhões de empregos sejam perdidos em todo mundo em virtude das políticas implementadas para combater a disseminação da COVID-19. Com agravo, Summer, Hoy e Ortiz-Juarez (2020) observam que o impacto da pandemia no curto prazo pode elevar os níveis de pobreza monetária no mundo, com uma estimativa de meio bilhão de pessoas reinseridas na pobreza. 
Samuel Façanha Câmara

Hermano José Batista de Carvalho

Felipe Gerhard

Vulnerabilidade socioeconômica da cidade de Fortaleza ao

Felipe Roberto da Silva

Covid-19: o epicentro da pandemia na região Nordeste do Brasil

Thiago Matheus de Paula

Desse modo, atendo-se às dimensões social e econômica, é importante entender o estado real de vulnerabilidade das áreas atingidas por surtos virais para, além de compreender os seus efeitos, identificar políticas e estratégias emergenciais que possam resolvê-las (MCLAFFERTY, 2010).

\section{FENÔMENOS PERVERSOS E OS SURTOS PANDÊMICOS}

Rittel e Webber (1973) foram os pioneiros no estudo dos fenômenos perversos. Para os autores, estes eventos se diferenciam dos problemas comuns, pois não há uma definição única para eles. Em outras palavras, para descrever um fenômeno perverso é necessário desenvolver um inventário exaustivo de todas as soluções possíveis. Não existem regras para solucionar estes problemas, sua solução não é previamente estruturada pelo fato de haver características únicas em relação a eventos semelhantes, ou seja, não existe uma solução correta, mas uma solução mais satisfatória ou suficiente (CROWLEY; HEAD, 2017; HEAD; ALFORD, 2015). Não é possível testar previamente a eficácia das soluções, isto é, após implementada a resolução, esta gerará consequências indeterminadas por um tempo ilimitado (KREUTER et al., 2004). Do mesmo modo, não existe um precedente ou similaridade; assim, um problema pode até soar familiar, mas não se pode ter certeza de que os detalhes de um problema podem ser substituídos por outros problemas semelhantes já tratados. Por fim, é socialmente complexo, pois as discrepâncias que envolvem um fenômeno perverso podem ser explicadas de inúmeras maneiras (RITTEL; WEBBER, 1973).

Para exemplificar estas diferenças entre problemas comuns e fenômenos perversos, Abreu e Andrade (2019) desenvolvem um problema racional e estrutural até a sua irracionalidade. Conforme os autores, em um desastre industrial, as instituições irão trabalhar em prol da mitigação dos impactos econômicos, ambientais e sociais. Porém, como há uma deficiência no sistema governamental quanto ao planejamento contingencial, preparo e mobilização de recursos, estes fenômenos podem se tornar complexos e imprevisíveis durante a aplicação das medidas (ABREU; ANDRADE, 2019).

As questões relacionadas aos fenômenos perversos estão diretamente associadas ao escopo governamental. Head e Alford (2015) ressaltam que as organizações governamentais conseguem se sobressair bem quando os serviços prestados já são padronizados e rotineiros, mas demonstram insegurança e ineficiência diante de serviços ou problemas fora do padrão. Reinicke, Benner e Witte (2001) explicam que esta dificuldade é devida à rigidez e inflexibilidade de modelos burocráticos convencionais.

Salienta-se que existe uma falsa disseminação de conhecimento de que a complexidade é um fator determinante na definição de fenômenos perversos, mas este motivo não é a característica primordial, uma vez que existem problemas matemáticos, químicos e físicos que são tecnicamente complexos, mas que podem ser claramente delineados e resolvidos por um sistema racional (KREUTER et al., 2004). Sendo assim, o que diferencia o fenômeno perverso dos demais é o dissenso que existe entre as partes interessadas quanto à sua origem, definição e solução, ou seja, são problemas de natureza única (RITTEL; WEBBER, 1973) e conhecimentos e experiências anteriores pouco ajudam.

Pandemias decorrentes de surtos virais como a COVID-19 podem entrar no escopo de fenômenos perversos, já que não apenas devastam meios de subsistência humana, mas também trazem diversas perturbações sociais, econômicas, ambientais físicas e biológicas (SANDS et al., 2016). Sendo assim, esta doença, por conta de suas especificidades, não permite que sejam estabelecidas regras claras para solucioná-la (XIANG, 2013; RITTEL; WEBBER, 1973). Nota-se, portanto, que o avanço da medicina traz melhorias técnicas e tecnológicas, as quais aliviam o efeito da letalidade de surtos virais. Contudo, estas doenças se expandem geograficamente, devido à dinâmica da estrutura econômica global, tornando as áreas e comunidades mais vulneráveis quanto a estes aspectos (SANDS et al., 2016). Diante dessas considerações, percebe-se que a resolução para estes problemas complexos possui interdependência e causas múltiplas associadas aos estressores causados pela infecção, desde 
o impacto na renda de cada indivíduo, até o colapso econômico, social, político, cultural e ambiental, necessitando, assim, de medidas satisfatórias na gestão pública que os minimizem (XIANG, 2013; KREUTER et al., 2004).

\section{METODOLOGIA}

Para alcançar o objetivo desta pesquisa, o qual visa identificar a vulnerabilidade associada à saúde e às dimensões social e econômica dos moradores dos bairros de Fortaleza quanto à pandemia de COVID-19, realizou-se um estudo exploratório, de natureza quantitativa, uma vez que se pretende amplificar a compreensão de um determinado problema (MALHOTRA, 2011).

De acordo com o decreto 14.498 de 2019, a cidade de Fortaleza possui oficialmente um total de 121 bairros, com a criação dos bairros "Aracapé" e "Novo Mondubim" em setembro do referido ano. Contudo, a não inserção desses bairros no estudo se deve à inexistência de dados censitários disponíveis para realização das análises perquiridas. Assim, a área de estudo desta pesquisa compreende 119 bairros de Fortaleza, capital do estado do Ceará, cuja população total, em 2019, foi estimada em 2,7 milhões de pessoas e uma densidade demográfica de aproximadamente 7,8 mil habitantes por quilômetro quadrado (IBGE, 2020). Ademais, neste estudo deu-se enfoque também a 11 Zonas Especiais de Interesse Social (ZEIS) já delimitadas em Fortaleza, sendo três destas estudadas pelo grupo de estudos das ZEIS da Universidade Estadual do Ceará (UECE), LabZEIS, pertencente ao Laboratório de Gestão de Cidades (LAGIC) presente nos Laboratórios Associados de Inovação e Sustentabilidade (LAIS).

Como estratégia de pesquisa, utilizou-se de levamento de dados secundários, obtidos na Prefeitura Municipal de Fortaleza (PMF, 2014, 2020), na Secretaria de Desenvolvimento Econômico de Fortaleza (SDE, 2020), no Instituto Brasileiro de Geografia e Estatística (IBGE, 2010) e na Relação Anual de Informações Sociais (RAIS, 2018), a qual contém a classificação de setores agrupados em classes estabelecidos pelo Código Nacional de Atividades Econômicas (CNAE) 2.0. Os dados coletados da Rais são referentes ao ano de 2018.

A análise dos dados foi dividida em três etapas. Na primeira, nomeada como Vulnerabilidade Ampla (VA), mensurou-se a vulnerabilidade social e econômica dos bairros. Nesta, foram utilizados dois parâmetros para o cálculo, a saber, número de pessoal ocupado e estimativa do valor da renda do pessoal ocupado; naquela, estabeleceu-se a relação entre as rendas dos mais pobres sobre os mais ricos. Ressalte-se que esta última trajetória empregada foi corrigida pela estimativa da renda de pessoas em idade economicamente ativa em situação de vulnerabilidade à pobreza (ATLAS, 2013). A partir destas informações, geraram-se as seguintes equações adaptadas de Câmara et al. (2020):

$$
\mathrm{VAE}_{\mathrm{PO}_{i}}=\mathrm{VPO}_{i} \quad \text { (Equação 1a) }
$$

Onde:

$\mathrm{VAE}_{\mathrm{POi}}=$ Vulnerabilidade ampla econômica por pessoal ocupado no respectivo ano e i-ésimo bairro de Fortaleza-Ce;

$\mathrm{VPO}_{\mathrm{i}}=$ vínculos ativos no respectivo ano e i-ésimo bairro;

$$
\left.\mathrm{VAE}_{\text {Renda }_{i}}=\left(\mathrm{VR}_{i} \cdot \mathrm{PO}_{i}\right) \quad \text { (Equação } 1 \mathrm{~b}\right)
$$

Onde:

VARendai $=$ Vulnerabilidade ampla econômica por renda anual no i-ésimo bairro de Fortaleza-CE;

$\mathrm{VR}_{\mathrm{i}}=$ Estimativa do valor da renda anual do i-ésimo bairro de Fortaleza-CE;

$\mathrm{PO}_{\mathrm{i}}=$ Pessoal ocupado total do respectivo bairro de Fortaleza-CE.

$$
\operatorname{VAS}_{i}=\left(\mathrm{VR}_{i} \cdot \mathrm{E}_{i}\right) \quad \text { (Equação 1c) }
$$


Samuel Façanha Câmara

Onde:

VASi $=$ Vulnerabilidade ampla social no i-ésimo bairro de Fortaleza-CE;

$V R_{i}=$ Estimativa da população economicamente ativa em situação de vulnerabilidade à pobreza do iésimo bairro (\% de pessoas com renda vulnerável a pobreza no i-ésimo bairro);

$\mathrm{E}_{\mathrm{j}}=$ Estimativa do valor da renda anual da população economicamente ativa vulnerável à pobreza do iésimo bairro de Fortaleza-CE.

Mensurada a VA, prosseguiu-se para a segunda etapa, a qual visava estimar a vulnerabilidade associada às características sociais e que se pode distinguir fortemente, neste caso, dos efeitos da pandemia da COVID-19 nos diferentes bairros da cidade, considerando-se um fator de correção territorial (FCT) para Vulnerabilidade Ampla, composto dos seguintes elementos: i) Mobilidade do bairro (M), uma vez que quanto mais pessoas transitam dentro do bairro ou entre bairros, maior seu nível de exposição; ii) Densidade demográfica (D), pois quanto maior o adensamento de pessoas maior a possibilidade de contagio; iii) Moradia (MO), considerando-se que quanto pior o ambiente onde as pessoas moram mais insalubre e anti-higiênico ele será, ampliando as chances de contágio e diminuindo a disposição das pessoas a ficarem em suas casas; iv) Violência (V), entendendo que quanto maior for a violência no bairro, mais isolado ele será do resto da cidade e para a COVID-19 isto diminuirá a vulnerabilidade; v) Nível Educacional (ED), visto que quanto maior for o nível educacional das pessoas no bairro, mais bem informados elas serão e mais corretamente seguirão as recomendações das autoridades de saúde; vi) Percentagem de Idosos maiores de 65 anos (ID), sabendo que quanto mais idosos maior risco de complicações sérias com a COVID-19. As vulnerabilidades que irão considerar estes fatores serão denominadas de Vulnerabilidade Territorial Econômica, tanto para a renda quanto para pessoal ocupado (VTERenda e VTEPO) e Vulnerabilidade Territorial Social (VTS), assim calculadas:

$$
\begin{aligned}
& \mathrm{VTE}_{\text {Renda }_{\mathrm{i}}}=\mathrm{VAE}_{\text {Renda } 1 \cdot \mathrm{FCT}_{\mathrm{i}}(\text { Equação } 2 \mathrm{a})} \\
& \mathrm{VTE}_{\mathrm{POi}}=\mathrm{VAE}_{\mathrm{PO}} \cdot \mathrm{FCT}_{\mathrm{i}}(\text { Equação } 2 \mathrm{~b}) \\
& \mathrm{VTS}=\mathrm{VAS} \cdot \mathrm{FCT}_{\mathrm{i}}(\text { Equação } 2 \mathrm{c}) \\
& \mathrm{FCT}_{\mathrm{i}}=\left(\mathrm{M}_{\mathrm{i}}+\mathrm{D}_{\mathrm{i}}+\mathrm{MO}_{\mathrm{i}}+\mathrm{V}_{\mathrm{i}}+\mathrm{ED}_{\mathrm{i}}+\mathrm{ID}_{\mathrm{i}}\right) \text { (Equação 3) }
\end{aligned}
$$

Onde:

$V T E_{\text {Rendai }}$ e $V T E_{P O i}=$ Vulnerabilidade territorial econômica para renda e para pessoal ocupado do iésimo bairro de Fortaleza-CE;

VAS = Vulnerabilidade territorial social para indivíduos economicamente ativos em condição de vulnerabilidade à pobreza do i-ésimo bairro de Fortaleza-CE;

$M_{i}, D_{i}, M_{i}, V_{i}, E D_{i}$ e ID $D_{i}$, Elementos do Fator de correção de Mobilidade, Densidade Demográfica, Moradia, Violência, Educação e Idosos acima de 65 anos, respectivamente, para o i-ésimo bairro.

O valor de FCT será: $0,25 \leq \mathrm{FCT}_{i} \leq 1,00$. Vale ressaltar que os valores dos elementos componentes do fator de correção territorial irão variar de acordo com seus quartis: quando o efeito sobre a vulnerabilidade é positivo, teremos que o Q1 = 0,041667; $Q 2=0,0833$; $Q 3=0,125$ e Q4 = 0,1667; e quando o efeito do fator for negativo, os valores dos quartis se invertem.

A terceira etapa da metodologia diz respeito ao cálculo de um nível mais específico da Vulnerabilidade dos bairros, saindo da Vulnerabilidade Territorial e incluindo a Vulnerabilidade associada às condições de Saúde. Novamente, parte-se do aspecto econômico tanto para renda quanto para pessoal ocupado (VSRendai $\mathrm{e}$ VSPOi); e social (VSS), para indivíduos economicamente ativos vulneráveis à pobreza. Neste caso, a VTERenda, VTEpo e VTS serão corrigidas por um fator de correção de saúde, composto dos seguintes elementos: i) quantidade de Postos de Saúde (PS), pois estes elementos básicos podem diferenciar e encaminhar as pessoas de forma mais correta durante maior nível de contágio e reduzir sua vulnerabilidade; ii) Quantidade de Unidades de Pronto Atendimento - UPA (UP), estas unidades 
Samuel Façanha Câmara

podem atender de forma mais imediata a população durante contágio mais intenso e reduzir sua vulnerabilidade; iii) Hospitais Públicos (HPub); e iv) Hospitais Privados (HP), por serem equipamentos dedicados a maior complexidade no atendimento, podem colocar pessoas mais acometidas em leitos e condições mais adequadas, reduzindo a vulnerabilidade do bairro; v) Proximidade de Hospitais de referência para COVID-19 (HR), porque esta proximidade pode ser crucial no atendimento mais rápido no caso de encaminhamentos dos casos mais graves; vi) Número das Equipes de Medicina da Família / população do bairro (MF), aceitando que quanto maior for esta relação mais cuidados de saúde as pessoas do bairro terão, reduzindo sua vulnerabilidade. Assim:

$$
\begin{aligned}
& \mathrm{VS}_{\text {Rendai }}=\mathrm{VTE}_{\text {Rendai }} \times \mathrm{FCS}_{\mathrm{i}} \text { (Equação 4a) } \\
& \mathrm{VS}_{\mathrm{POi}}=\mathrm{VTE}_{\mathrm{POi}} \times \mathrm{FCS}_{\mathrm{i}} \text { (Equação } 4 \mathrm{~b} \text { ) } \\
& \text { VSS }=\text { VTS } \times \text { FCS }_{\mathrm{i}} \text { (Equação 4c) } \\
& \mathrm{FCS}_{\mathrm{i}}=\left(\mathrm{PS}_{\mathrm{i}}+\mathrm{UP}_{\mathrm{i}}+\mathrm{HPub}_{\mathrm{i}}+\mathrm{HP}+\mathrm{HR}_{\mathrm{i}}+\mathrm{MF}_{\mathrm{i}}\right) \text { (Equação 5) }
\end{aligned}
$$

Onde:

$P S_{i}, U P_{i}, H P u b_{i}, H P, H R_{i}, M_{i}=$ Elementos do Fator de correção para Postos de Saúde, Quantidade de UPAs, Quantidades de Hospitais Públicos, Proximidade de Hospitais de referência e Quantidade de Equipes de Saúde da Família, respectivamente para o i-ésimo bairro.

O valor de FCS será: $0,25 \leq F C S_{i} \leq 1,00$. Salienta-se que os valores dos elementos componentes do fator de correção de saúde irão variar de acordo com seus quartis: quando o efeito sobre a vulnerabilidade é positivo, teremos que o Q1 = 0,041667; $\mathrm{Q} 2=0,0833$; $\mathrm{Q} 3=0,125$ e Q4 = 0,1667 e quando o efeito de fator for negativo, os valores dos quartis se invertem.

$\mathrm{Na}$ última etapa da pesquisa, aplicaram-se os mesmos níveis de vulnerabilidade nas onze Zonas Especiais de Interesse Social (ZEIS). É importante salientar que, para três destas (ZEIS Pirambu, Lagamar e Moura Brasil), foram utilizados dados coletados pela pesquisa de campo, realizada pelo grupo de estudos das ZEIS LabZEIS, vinculado ao LAGIC. Quanto às demais, foi estimado o perímetro total das ZEIS e seu percentual nos respectivos bairros nos quais estão inseridas. Dessa forma, calculou-se a média ponderada da vulnerabilidade destes bairros, a partir do percentual de participação territorial das ZEIS. Finalmente, ressalta-se que os dados referentes aos índices de vulnerabilidade foram tratados no editor de planilhas Excel e exportados para a plataforma de mapeamento geográfico Qgis 3.14.1, na qual as localidades foram georreferenciadas e os mapas editados.

\section{ANÁLISE DOS RESULTADOS}

A seguir, estão apresentadas as análises de vulnerabilidade social e econômica divididas em três etapas: Vulnerabilidade Socioeconômica Ampla (VA), Territorial (VT) e Saúde (VS). Os limites georreferencias das análises dos dados foram divididas, isto é, primeiro foram expostos os resultados dos bairros e, posteriormente, apresentaram-se os achados das ZEIS.

\section{Vulnerabilidade ampla}

Problemas com agentes patogênicos em grandes proporções, como surtos pandêmicos, podem ser avaliados em diferentes escalas e percepções. Existe não apenas uma perda real biológica, mas também um impacto social e econômico para as comunidades ou sociedades afetadas, tendo em vista que estas implicações podem se dar de forma gradativa, isto é, gerando consequências tanto em curto como em longo prazo (LI et al., 2020; NELSON et al., 2018; MCLAFFERTY, 2010). Sabendo disso, partiu-se de diferentes trajetórias para se avaliar estas perdas. Em Vulnerabilidade Ampla Econômica (VAE), o olhar analítico foi sobre o aspecto econômico de cada bairro. Assim, na Figura 1, pode-se observar os bairros mais suscetíveis ao impacto. 
Samuel Façanha Câmara

Hermano José Batista de Carvalho

Felipe Gerhard

Vulnerabilidade socioeconômica da cidade de Fortaleza ao

Felipe Roberto da Silva

Covid-19: o epicentro da pandemia na região Nordeste do Brasil

Thiago Matheus de Paula

Figura 1 - Fortaleza (CE): Vulnerabilidade Ampla Econômica e Social por bairros de Fortaleza, 2020. A. Renda; B. Pessoal ocupado; C. Renda vulnerável.
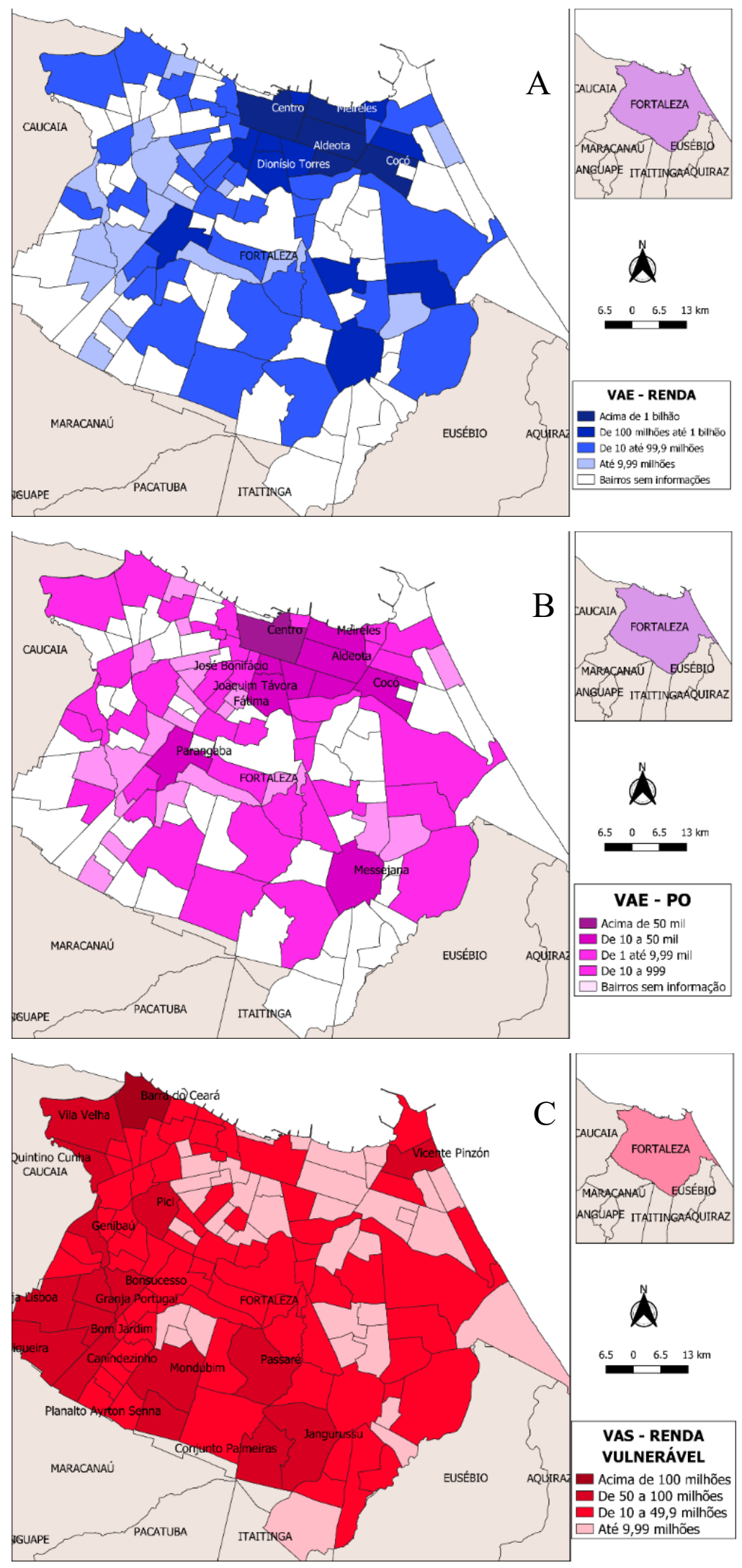

Fonte - elaboração dos autores com Qgis. 
Samuel Façanha Câmara

Hermano José Batista de Carvalho

Felipe Gerhard

Vulnerabilidade socioeconômica da cidade de Fortaleza ao

Felipe Roberto da Silva

Covid-19: o epicentro da pandemia na região Nordeste do Brasil

Thiago Matheus de Paula

Pela mensuração da VAE na trajetória da estimativa da renda nos bairros, percebe-se (Figura 1A) que a área mais suscetível a este aspecto é a Aldeota com um índice de, aproximadamente, $R \$ 2,9$ bilhões, seguida de Meireles, Centro, Cocó e Dionísio Torres. Todos estes bairros apresentaram índices acima de $R \$ 1$ bilhão, o que representa uma possível perda de $R \$ 10,4$ bilhões para o município de Fortaleza, em 2020, considerando o somatório desses bairros. Ressalta-se que estas áreas estão entre os sete bairros de maiores taxas de empregabilidade e, consequentemente, maiores rendas por indivíduo.

Analisando a VAE pelo parâmetro de pessoal ocupado (Figura 1B), novamente não há grandes mudanças, alterando-se apenas a ordem dos bairros. O Centro de Fortaleza agora ocupa a primeira colocação com um VEA de mais de 91,2 mil (19\%) vínculos formais ativos. Além desse, Aldeota, Meireles, Dionísio Torres e Cocó seguem com índices de 50,6, 35,7, 23,6 e 22 mil vínculos, respectivamente. No bairro Centro, o setor de atividade econômica mais presente é a Administração Pública, a qual concentra $18,3 \%$ do total de pessoal ocupado, lembrando que este setor está bem abaixo na linha de vulnerabilidade e é um dos menos afetados no atual cenário do estado (ABRASEL, 2020). Porém, existe também uma grande concentração de comércio varejista na região, com destaque para lojas de artigo de vestuário e acessórios que somam 625 estabelecimentos formais $(9,4 \%)$, gerando um total de 2,7 vínculos empregatícios (3\%), além de bares, restaurantes, lanchonetes, casas de sucos e similares, que juntas somam 347 estabelecimentos e 3.293 vínculos (SDE, 2019).

Em outros contextos econômicos, estes setores sofreram um grande impacto de surtos virais anteriores, como na Síndrome Respiratória Aguda Grave (SARS) (SANDS et al., 2016; SIU; WONG, 2004; MCKERCHER, 2003). Entre os setores, vale destacar o turismo e hotelaria, o qual sofreu queda nos serviços durante a pandemia por consequência do medo e insegurança instalados nos países em que a transmissibilidade foi crescente (SANDS et al., 2016; SIU; WONG, 2004; MCKERCHER, 2003). Destaca-se que na cidade de Fortaleza, no ano de 2018 , foram pagos $R \$ 7,2$ milhões em salários neste segmento (RAIS, 2018).

$\mathrm{Na}$ Figura 1C são dados os estratos de Vulnerabilidade Ampla Social dos bairros. Assim, percebe-se que o maior índice concentra na Barra do Ceará, cuja renda vulnerável ultrapassa os $R \$ 106,4$ milhões. Saliente-se que na relação entre a renda dos mais ricos e mais pobres desse bairro, $48 \%$ dos salários ficam com a classe mais pobre. Em seguida, vêm Mondubim, Granja Lisboa, Vila Velha e Canindezinho, cujas rendas vulneráveis são $11 \%, 30 \%, 35 \%$ e $55 \%$ menor que a primeira área, respectivamente. Dos cinco primeiros bairros, destacam-se Canindezinho, onde $84 \%$ dos rendimentos concentram-se na classe dos mais pobres e Granja Lisboa, cujo percentual é de aproximadamente $72 \%$.

$\mathrm{Na}$ dinâmica espaço-temporal das infecções, aqueles marginalizados socialmente acabam sendo os mais prejudicados, haja vista estarem em pior condição não só econômica, mas também alimentar e imunológica (NEIDERUD 2015; MCLAFFERTY, 2010). Esta realidade pode ser observada com a confirmação dos casos de coronavírus por bairros de Fortaleza, até 18 de agosto de 2020. Os bairros Barra do Ceará e Vila Velha eram as áreas com maiores números de óbitos por coronavírus à data, com 124 e 109 pessoas, respectivamente (SMS, 2020), apesar do bairro Meireles concentrar a maioria dos infectados (1750 pessoas).

\section{Vulnerabilidade territorial}

Lins-de-Barros (2017) frisa que as consequências geradas pela ameaça à região podem variar, a depender do seu nível de exposição e suscetibilidade. Dessa forma, é necessária a compreensão dos bairros na análise pelos seus principais indicadores, como densidade demográfica, violência, educação, IDH e tamanho da população. Ademais, tratando-se de um surto pandêmico, a mobilidade da área torna-se uma variável essencial, uma vez que esta impacta na disseminação do vírus e no tamanho do contágio, devendo ser considerada para a mensuração da vulnerabilidade (KRAEMER et al., 2020). Dessa forma, a Vulnerabilidade Territorial Econômica e Social podem ser observados na Figura 2. 
Samuel Façanha Câmara

Hermano José Batista de Carvalho

Felipe Gerhard

Vulnerabilidade socioeconômica da cidade de Fortaleza ao

Felipe Roberto da Silva

Covid-19: o epicentro da pandemia na região Nordeste do Brasil

Thiago Matheus de Paula

Figura 2 - Fortaleza (CE): Índices de Vulnerabilidade Territorial Econômico e Social por bairros, 2020. A. Renda; B. Pessoal ocupado; C. Renda vulnerável.
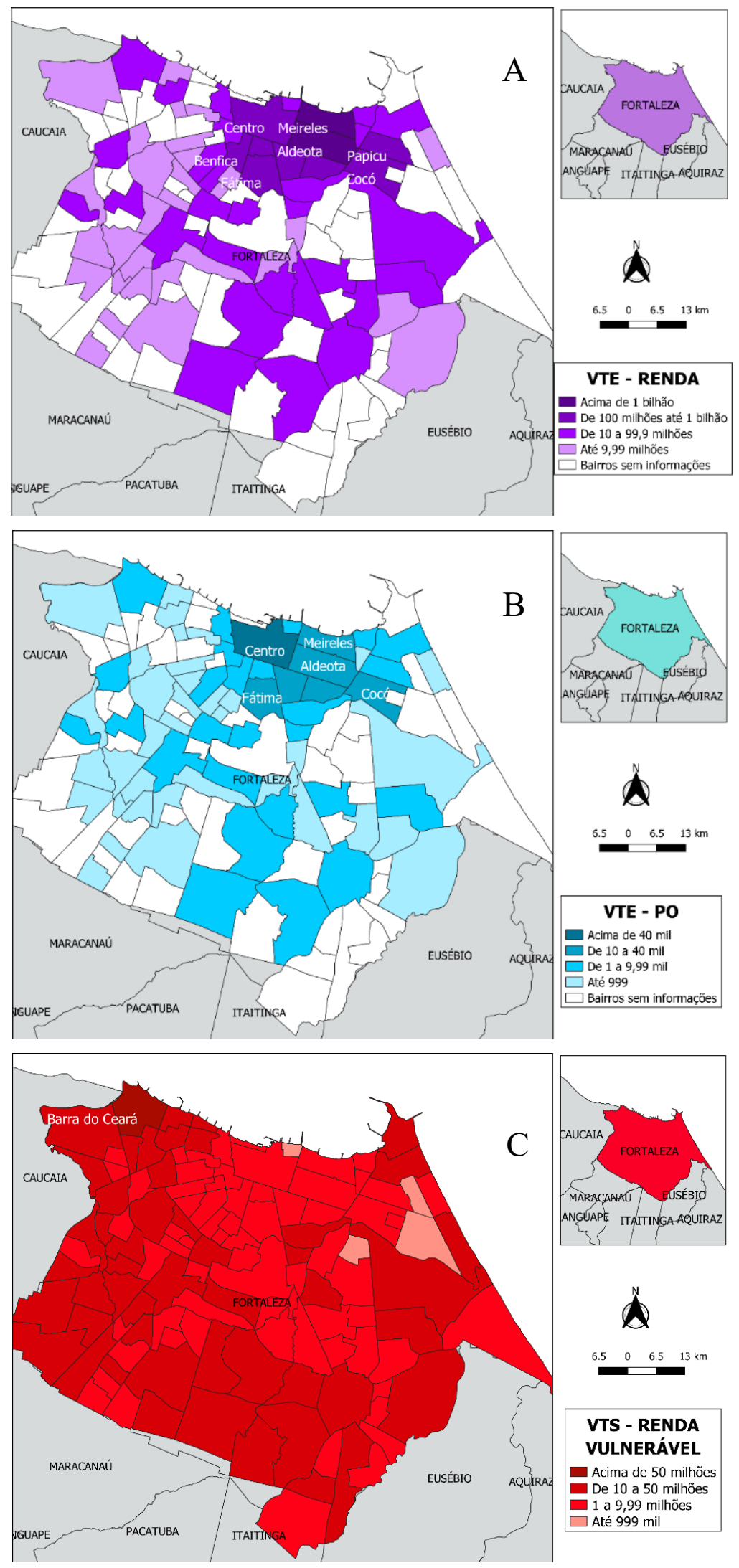

Fonte - Elaboração dos autores com Qgis. 
Samuel Façanha Câmara

Hermano José Batista de Carvalho

Felipe Gerhard

Vulnerabilidade socioeconômica da cidade de Fortaleza ao

Felipe Roberto da Silva

Covid-19: o epicentro da pandemia na região Nordeste do Brasil

Thiago Matheus de Paula

Quanto ao cálculo da Vulnerabilidade Territorial Econômica, na primeira mensuração, utilizando como parâmetro a renda dos bairros (Figura 2A), Meireles inverte a colocação com Aldeota, cujos índices foram $R \$ 1,5$ e $R \$ 1,4$ bilhões, respectivamente. Centro, Cocó e Dionísio Torres seguem entre as cinco primeiras áreas, cujos índices somam 2, bilhões, evidenciando a suscetibilidade à perda de rendimento das pessoas que trabalham nesses bairros.

Por outro lado, tendo como parâmetro o pessoal ocupado (Figura 2B), o Centro de Fortaleza é a região de maior Vulnerabilidade Territorial Econômica, com um índice de 42,3 mil vínculos ativos. Os bairros que seguem são Aldeota, Meireles, Joaquim Távora e Cocó, os quais representam menos da metade da vulnerabilidade do Centro, salvo Aldeota que concentra um índice de 23,4 mil. Salienta-se que o centro é o segundo bairro com maior mobilidade urbana, perdendo apenas para Arraial Moura Brasil.

Estudos anteriores (e.g. KRAEMER et al., 2020, LINS-DE-BARROS, 2017; PAVEGLIO et al., 2016) apontam uma relação direta entre o grau de vulnerabilidade e as variáveis mobilidade humana e densidade demográfica, isto é, a grande concentração da população e o fluxo urbano elevam o nível de exposição e suscetibilidade das regiões ao perigo. Sendo assim, bairros como Centro, Aldeota, Meireles e Dionísio Torres, os quais estão entre as quinze maiores mobilidades urbanas do município, pontuaram um peso maior no indicador.

Na Figura 2C, tem-se a Vulnerabilidade Territorial Social, cujo maior índice novamente foi do bairro da Barra do Ceará, com uma renda vulnerável de $\mathrm{R} \$ 72,2$ milhões. Em segundo lugar ficou a Granja Lisboa, com uma renda de 43,9 milhões e em terceiro Genibaú, com R\$ 42,6 milhões. Finalmente, a quarta e a quinta colocação ficaram com Quintino Cunha e Vila Velha, com 37,7 e 36,5 milhões, respectivamente. Curiosamente, três destes bairros estão entre as 15 regiões de maior densidade em Fortaleza, ou seja, Quintino Cunha tem uma densidade de 22,1 mil pessoas por quilômetro quadrado, enquanto Genibaú e Barra do Ceará, 18,6 e 16,9 mil, respectivamente. Além disso, ressalta-se que o bairro Granja Lisboa tem um dos 20 piores desempenhos na educação, indicando uma maior vulnerabilidade neste aspecto (PMF, 2020).

\section{Vulnerabilidade associadas às condições de saúde}

Em continuidade às análises de vulnerabilidades socioeconômicas, outra dimensão relevante para compreensão das consequências geradas pela presença de patógenos em comunidades, sociedades ou territórios é o grau de privação individual a serviços de saneamento e saúde adequados (NEIDERUD, 2015; MCLAFFERY, 2010). Neste âmbito, a dimensão da saúde, abordada através do acesso a centros de atendimento, como número de posto de saúde, UPAs e Hospitais, e pelo números de equipes de medicina da família, revela-se de suma importância para reduzir os níveis de vulnerabilidade, uma vez que possibilita aos indivíduos do bairro acesso aos primeiros cuidados e informações de saúde.

Em relação aos valores da Vulnerabilidade Econômica associada às condições de Saúde, a qual utilizou em sua primeira mensuração a renda dos bairros (Figura $3 \mathrm{~A}$ ), novamente Meireles e Aldeota apresentaram-se como mais vulneráveis, com um total de $\mathrm{R} \$ 1,6$ e R\$1,3 bilhões, respectivamente. Em seguida, os bairros do Cocó, Centro e Dionísio Torres encontram-se entre as cinco áreas mais impactadas, cujos índices somam um total de $\mathrm{R} \$ 2,4$ bilhões, o qual simboliza a suscetibilidade à perda de rendimento das pessoas que trabalham nos respectivos bairros.

Quando analisado sob o parâmetro de número de pessoal ocupado (Figura 3B), a disposição dos bairros se assemelha aos resultados da Vulnerabilidade Territorial Econômica, em que o Centro de Fortaleza lidera o ranking de bairro mais vulnerável, com um índice de 33.862 vínculos formais ativos. Os bairros que o seguem são Aldeota (23.476), Meireles (22.428), Cocó (15.128) e Joaquim Távora (14.960), compreendendo os cinco bairros de maior Vulnerabilidade de Pessoal Ocupado associada às condições de Saúde. Dessa forma, visto que esses bairros concatenam grande parte dos vínculos empregatícios da cidade, os índices acima revelam o elevado nível de exposição e suscetibilidade aos quais essas regiões estão expostas, constituindo um total de 220.901 mil demissões que potencialmente poderão ocorrer nos estabelecimentos concentrados nesses bairros. Isto posto, os resultados da mensuração da VS podem ser visualizados na Figura $3(A, B$ e $C)$ a seguir. 
Samuel Façanha Câmara

Hermano José Batista de Carvalho

Felipe Gerhard

Vulnerabilidade socioeconômica da cidade de Fortaleza ao

Felipe Roberto da Silva

Covid-19: o epicentro da pandemia na região Nordeste do Brasil

Thiago Matheus de Paula

Figura 3 - Fortaleza (CE): Índices de Vulnerabilidade associado às condições de Saúde por bairros, 2020. A. Renda; B. Pessoal ocupado; C. Renda vulnerável.
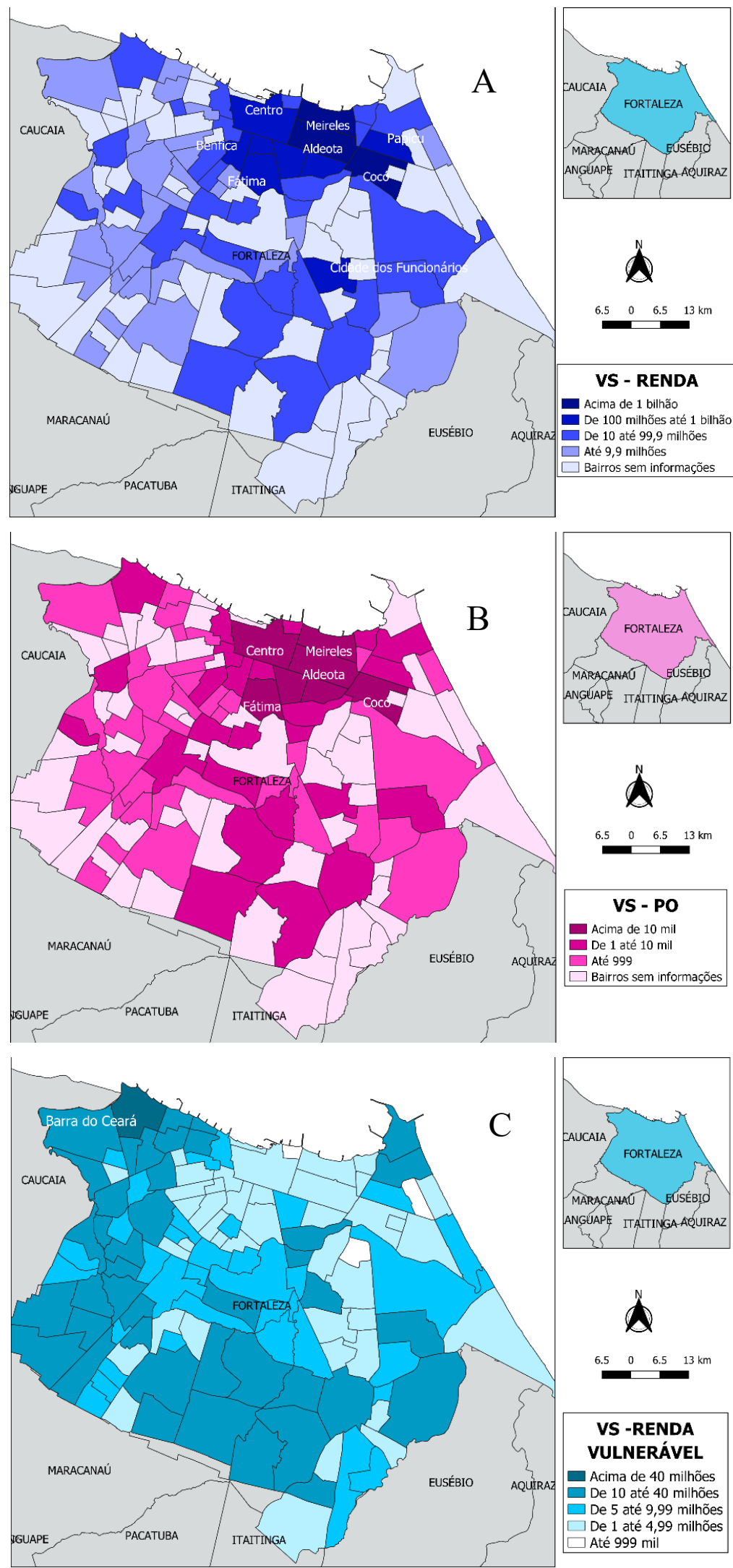

Fonte - Elaboração dos autores com Qgis. 
Quanto à Vulnerabilidade Social associada às condições de Saúde (Figura $3 C$ ), o maior índice foi, novamente, apresentado pelo bairro da Barra do Ceará, com uma renda vulnerável de $R \$ 46,9$ milhões. Em seguida, a Granja Lisboa apresentou o segundo maior índice, com uma renda suscetível à perda estimada em $\mathrm{R} \$ 37,2$ milhões; logo após, o bairro Genibaú, com 36,2 milhões, seguido na quarta e quinta colocação pelos bairros Quintino Cunha e Pici, com uma renda vulnerável estimada de 32,0 e 30,7 milhões, respectivamente.

Para esta última medida, observa-se que as condições socioeconômicas, pessoas vulneráveis à pobreza, condições de moradia, educação e outros parâmetros analisados pela Vulnerabilidade Territorial, quando associadas às privações de acesso a serviços essenciais de saúde elevam a exposição de regiões com maiores desigualdades sociais. Ademais, ressalta-se que, para esses indicadores de saúde, todos os bairros encontraram-se nos quartis mais elevados - com exceção para os parâmetros de Postos de Saúde e número de Agentes de Saúde da Família -, indicando baixa oferta de serviços de saúde à comunidade do bairro, o que fez com que tivessem um maior peso destas variáveis no cálculo da vulnerabilidade. Tais condições, em consonância com a literatura (NEIDERUD, 2015; MADHAV et al., 2017), sugerem que grupos sociais mais vulneráveis à pobreza em associação a condições de privações sociais podem enfrentar maiores riscos durante uma pandemia.

\section{Zonas Especiais de Interesse Social (ZEIS)}

Em 2019, a cidade de Fortaleza tinha a população estimada de 2,7 milhões de habitantes, distribuídos em 121 bairros, os quais são agrupados geograficamente em sete Regionais Executivas, ocupando um território de aproximadamente 312 mil metros quadrados (IBGE, 2020). Além disso, os dados econômicos demostram que o salário médio se encontrava em 2,7 salários mínimos, em 2017, oriundos de 841 mil pessoas ocupadas, significando $32 \%$ da população total. No mesmo ano, $36,9 \%$ dos domicílios da cidade tinham rendimentos de meio salário mínimo, enquanto a renda per capita representava R\$24,4 mil, o que justifica o alto Coeficiente Gini de 0,619 (IPLANFOR, 2016).

Com efeito, a concentração de renda da cidade de Fortaleza é demonstrada na sua própria divisão geográfica (Figura 4), pois os dez bairros mais ricos, onde residem $7 \%$ da população, concentram uma riqueza superior aos 44 bairros de menor renda, onde se situam $49 \%$ da população da capital cearense. Destaque-se, nesta concentração, o bairro do Meireles (Zona Leste), cuja renda média é superior em 15,3 vezes ao do bairro do Conjunto Palmeiras, considerado o mais pobre (IPECE, 2012).

Figura 4 - Fortaleza (CE): Caracterização dos Bairros de Fortaleza por Renda, 2020.

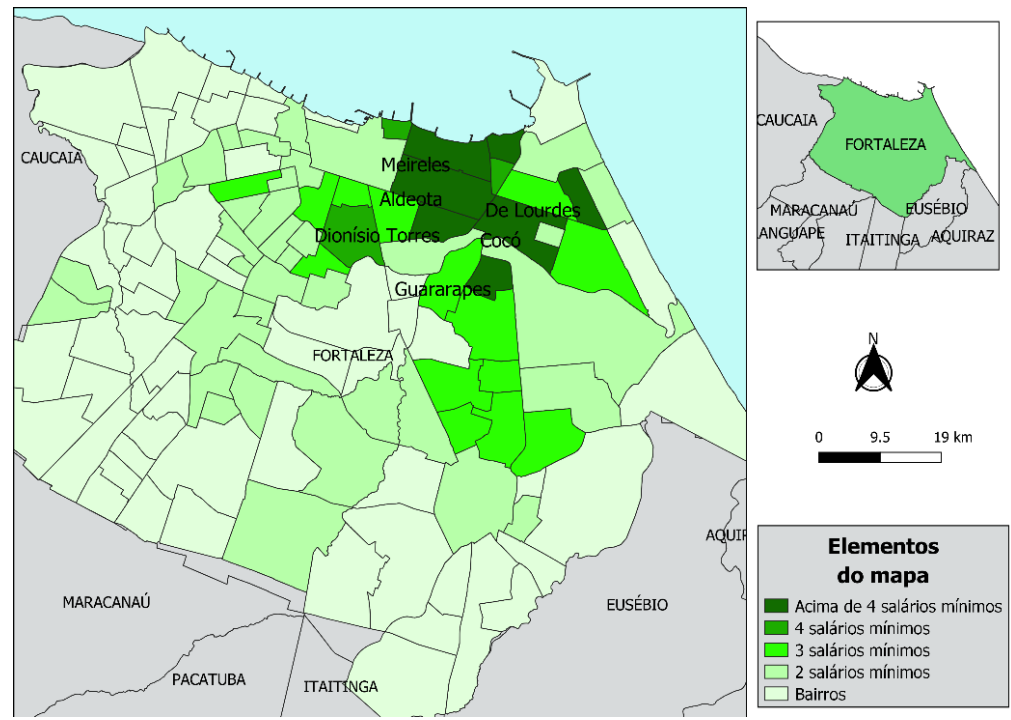

Fonte - Elaboração dos autores com base nos dados de Fortaleza 2040 (PMF, 2020).

É nesse contexto que se concretiza, em 2009, o Plano Diretor Participativo (PDPFor), o qual define 131 Zonas Especiais de Interesse Social (ZEIS) no município de Fortaleza. Estas áreas representam partes

Caminhos de Geografia Uberlândia-MG $\quad$ v. 22, n. $80 \quad$ abr./2021 $\quad$ p. 133-152 Página 145


Samuel Façanha Câmara Hermano José Batista de Carvalho Felipe Gerhard

Vulnerabilidade socioeconômica da cidade de Fortaleza ao Felipe Roberto da Silva

do território da cidade, situadas seja em propriedades públicas ou privadas, que devem ser alvos prioritários de ações de regularização urbanística e fundiária por serem locais onde residem pessoas com baixa renda que, pelo tempo que assentadas, podem ser consideradas consolidadas. Nessas áreas devem ser desenvolvidos programas habitacionais e de estímulo ao mercado popular. Por sua natureza especial, essas localidades deverão ter uma legislação urbanística própria, com critérios de edificação, parcelamento e uso e ocupação do solo, respeitando a situação consolidada (IPLANFOR, 2016).

A seguir, apresentam-se as análises dos dados e resultados das Zonas Especiais de Interesse Social (ZEIS) presentes no município de Fortaleza.

\section{Vulnerabilidades Social e Econômica das ZEIS}

Das 131 Zonas Especiais de Interesse Social de Fortaleza, existem algumas áreas prioritárias, a saber: Zeis Mucuripe, Serviluz, Moura Brasil, Pirambu, Lagamar, Poço da Draga, Bom Jardim, Pici, Dionísio Torres e Praia do Futuro. Ressalta-se que esta última é composta por duas ZEIS que se concentram no bairro. Com efeito, mensuraram-se os índices de vulnerabilidade destas 11 áreas e os resultados podem ser observados na Figura 5 a seguir.

Figura 5 - Fortaleza (CE): Índices de Vulnerabilidade, 2020. A. VAE Renda; B. VAE Pessoal ocupado; C. VAS Renda vulnerável; D. VTE Renda; E. VTE Pessoal ocupado; F. VTS Renda vulnerável; G. VS Renda; H. VS Pessoal ocupado; I. VSS Renda vulnerável.
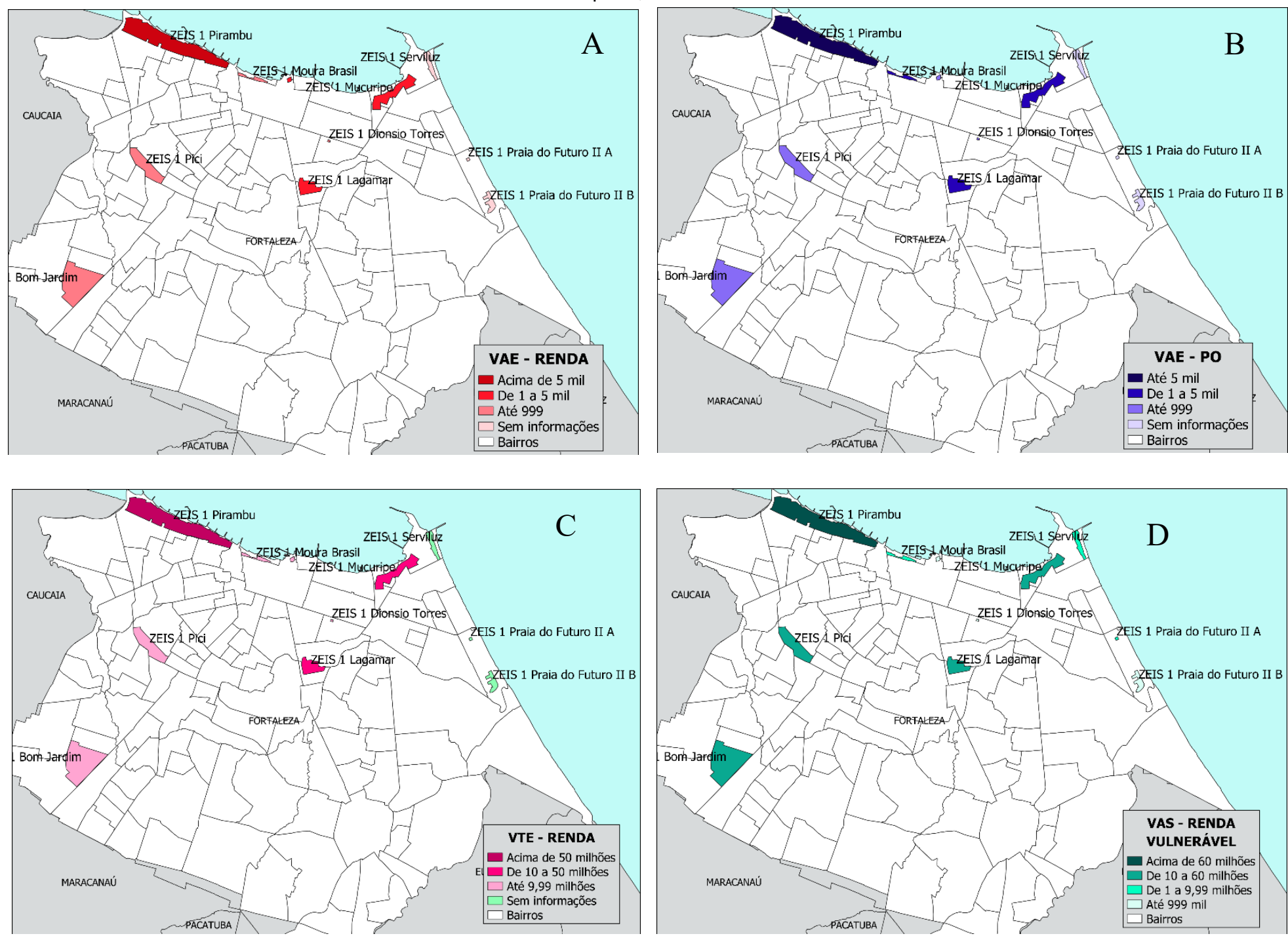
Samuel Façanha Câmara

Hermano José Batista de Carvalho

Felipe Gerhard

Vulnerabilidade socioeconômica da cidade de Fortaleza ao

Felipe Roberto da Silva

Covid-19: o epicentro da pandemia na região Nordeste do Brasil

Thiago Matheus de Paula
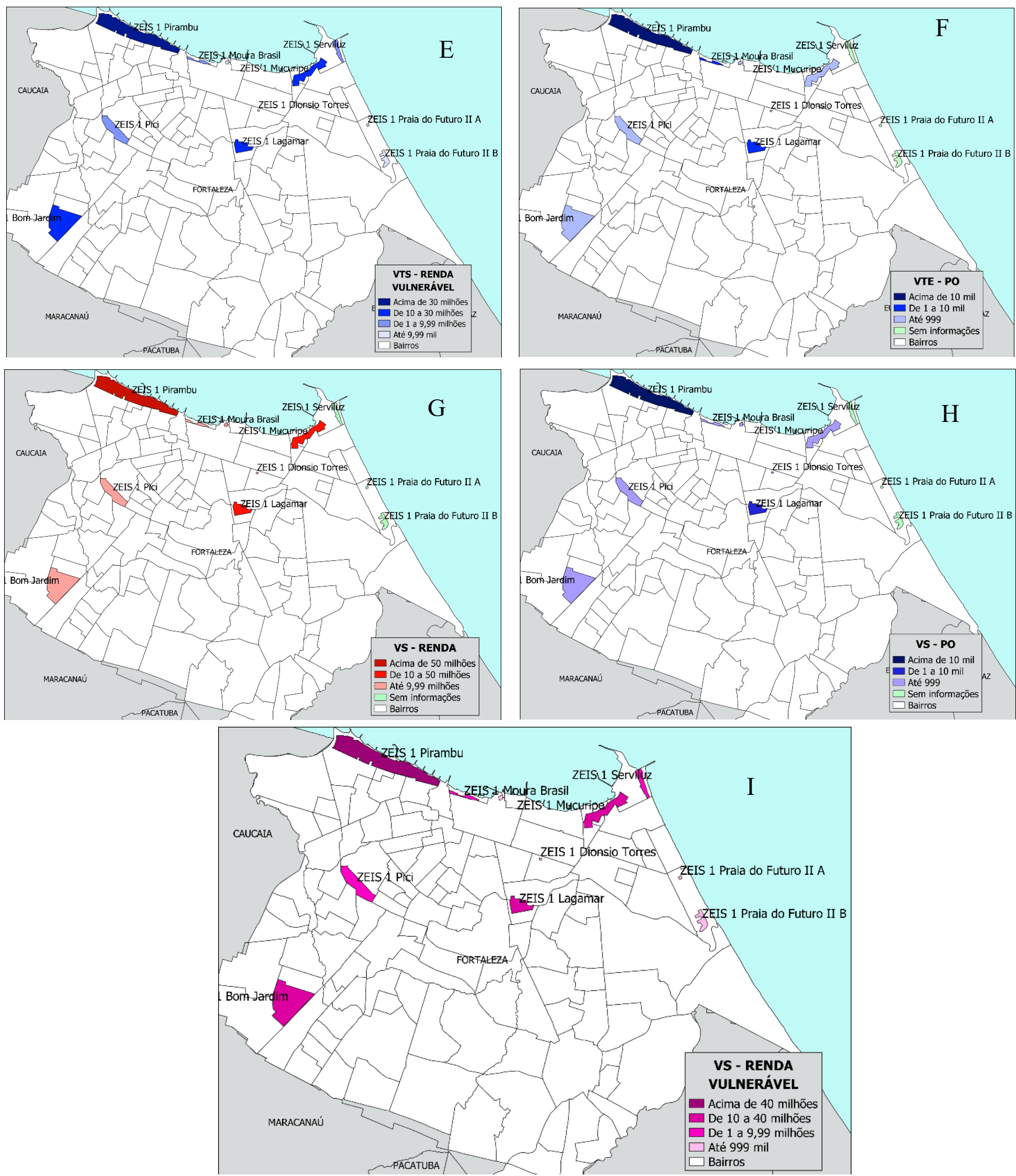

Fonte - Elaboração dos autores com Qgis. 
Samuel Façanha Câmara

Hermano José Batista de Carvalho

Felipe Gerhard

Vulnerabilidade socioeconômica da cidade de Fortaleza ao

Felipe Roberto da Silva

Covid-19: o epicentro da pandemia na região Nordeste do Brasil

Thiago Matheus de Paula

Economicamente, a ZEIS Pirambu (cuja área integra, principalmente, os bairros de Pirambu, Cristo Redentor e Barra do Ceará) é potencialmente a região mais vulnerável dentre as zonas especiais. Como se pode observar na Figura 5A e 5B, os índices de Vulnerabilidade Ampla Econômica desta ZEIS foram, aproximadamente, 38 mil vínculos ativos e uma renda suscetível a perdas de $R \$ 272,5$ milhões. Quando acrescentados os indicadores característicos dos bairros, a Vulnerabilidade Territorial chegou a quase 19 mil, para pessoal ocupado, e concentração de $R \$ 136$ milhões, na renda. Vale ressaltar que esta região é a terceira de maior densidade demográfica da cidade, com cerca de 37,5 pessoas por quilômetro quadrado, e uma grande concentração de pessoas acima de 65 anos na região, representando $6,6 \%$ da população. Do mesmo modo, possui um dos maiores índices de violência do município, com aproximadamente 35,6 homicídios por 100 mil habitantes. Ademais, quanto à Vulnerabilidade associada às condições de saúde, salienta-se que a ZEIS Pirambu obteve os maiores quartis, uma vez que há uma baixa oferta de serviços de saúde na região, salvo os postos de saúde, os quais somam cinco espalhados pela comunidade.

A mesma situação se observa na vulnerabilidade social, onde novamente o Pirambu se destaca. Com uma renda vulnerável ampla e territorial de $\mathrm{R} \$ 150,7$ e $\mathrm{R} \$ 75,3$ milhões, respectivamente, nesta zona, mais da metade do rendimento da população (52\%) concentra-se na classe mais pobre. Assim, analisando o perfil dos três principais bairros onde esta ZEIS se encontra, o percentual de indivíduos que estão abaixo da linha de pobreza é significativamente alto. Conforme o Instituto de Planejamento de Fortaleza (IPLANFOR, 2015), o Pirambu é o oitavo bairro de Fortaleza com maior percentual de sua população vivendo em situação de extrema pobreza, com um total de 1.831 indivíduos $(10,30 \%)$, seguido por Barra do Ceará $(6,64 \%)$ e Cristo Redentor $(5,99 \%)$, os quais apresentam taxas relativamente altas, 4.808 e 1.601 pessoas vivendo abaixo da linha de pobreza, respectivamente. Para efeitos comparativos, a cidade de Fortaleza, de forma geral, apresenta $5,46 \%$ de sua população nestas condições (IPLANFOR, 2015).

Do mesmo modo, destaca-se como zona de alta vulnerabilidade socioeconômica a ZEIS Lagamar, região ranqueada como a segunda mais vulnerável quanto ao pessoal ocupado, totalizando mais de 3,4 mil vínculos formais ativos. Por outro lado, o Mucuripe é a segunda mais suscetível nos indicadores de renda, com uma vulnerabilidade de $\mathrm{R} \$ 64,7$ milhões somente em VAE (Figura 5A). Finalmente, Bom Jardim vem logo após o Pirambu no ranking das ZEIS mais vulneráveis socialmente, com renda vulnerável ampla e territorial de $\mathrm{R} \$ 57,4$ e $\mathrm{R} \$ 40,7$ milhões, respectivamente.

\section{CONSIDERAÇÕES FINAIS}

Utilizando-se por base a mensuração e a interrelação dos indicadores de vulnerabilidade socioeconômica desenvolvidos, os principais achados da pesquisa podem ser divididos em três pontos centrais. Primeiramente, percebe-se uma nítida divisão econômica e social na geografia de Fortaleza, o que era de se esperar pela constituição dos bairros periféricos nas metrópoles brasileiras. Essa constatação salienta o abismo existente entre bairros ricos e pobres em Fortaleza. Enquanto os mais ricos se destacam na análise por apresentarem uma alta vulnerabilidade econômica, ligada à produção dos bairros, os mais pobres se destacam pela alta vulnerabilidade social, o que se mantém quando as condições territoriais de saúde são consideradas. Apesar deste resultado ser esperado, ficam claras as magnitudes de riscos associadas de forma comparativa entre estes bairros, posicionando bairros como a Barra do Ceará na perspectiva de alerta para as políticas públicas que devem se apoiar no estímulo à sua resiliência, considerando suas condições de território e de beneficiários dos serviços de saúde.

A relação entre bairros ricos e pobres na capital introduz o nosso segundo ponto de discussão. Enquanto fenômeno perverso, a disseminação da pandemia varia de acordo com as particularidades sociais-econômicas, ambientais e físicas de uma determinada realidade territorial. A sua complexa configuração suscita a possibilidade de existência de crises pandêmicas distintas dentro de um mesmo ambiente macrossocial. Tal complexidade traduz-se não apenas pelos períodos de avanço irregular da doença, iniciando-se predominantemente nos bairros ricos da capital, mas pela necessidade de estratégias completamente diferentes para impedir o seu avanço. O enfrentamento da disseminação deve ocorrer de formas diferentes entre os distintos contextos sociais, haja visto sua maior perversidade nos bairros com maiores privações sociais. O déficit no atendimento das necessidades básicas e a insuficiência dos programas de auxílio governamentais para socorro das famílias mais pobres geram problemas que intensificam os impactos da pandemia. A pandemia escancarou ainda mais o abismo 
Samuel Façanha Câmara

Hermano José Batista de Carvalho

Felipe Gerhard

Vulnerabilidade socioeconômica da cidade de Fortaleza ao

Felipe Roberto da Silva

Covid-19: o epicentro da pandemia na região Nordeste do Brasil

Thiago Matheus de Paula

de desigualdade que separa bairros ricos e pobres, sendo premente ao seu combate ações não somente necessárias, como ações higiênicas, mas também medidas suficientes e satisfatórias que minimizem as vulnerabilidades sociais.

Em terceiro lugar, as ZEIS representam um contexto contraditório dentro da realidade pandêmica projetada para Fortaleza. O coração dessas zonas geralmente se caracteriza pelo binômio pobreza e presença de barreiras sociais e econômicas de entrada. Apesar de não figurarem no ranking das regiões mais vulneráveis em todos os indicadores, essas particularidades associadas a elas criam um problema: a dificuldade de contenção da infecção e tratamento dos infectados caso a disseminação da doença atinja suas famílias. Essa é uma preocupação adicional relacionada à realidade de combate da Covid-19 em contextos de pobreza e precariedade da estrutura pública. Dessa forma, essas zonas merecem atenção especial por parte da Prefeitura e Governo do Estado para que, uma vez atingida pela pandemia, sua população não sofra ainda mais com os isolamentos socioeconômicos enfrentados cotidianamente.

Em suas limitações, este estudo utiliza-se de bases de dados das instâncias de governo no Brasil, como a RAIS, cujas informações são referentes ao mercado de trabalho formal da cidade de Fortaleza no ano de 2018, o que impossibilitou ver o real cenário da economia como um todo, por ausência de dados sobre a economia informal. Nesse aspecto, os resultados apresentados são mais indicativos. Do mesmo modo, ressalta-se como limitação do estudo a disparidade dos dados do censo do IBGE (2010) especialmente em relação à densidade populacional de aglomerados subnormais e os perfis de renda de suas famílias. A pesquisa realizada com dados coletados nas Zonas Especiais de Interesse Social, em 2019, revela que os dados do censo 2010 podem não apenas estar desatualizados, como não representar com fidedignidade esses aspectos. Para pesquisas futuras, portanto, sugere-se avaliar a vulnerabilidade em outras capitais ou estados epicentros da pandemia de COVID-19, considerando-se as três etapas analisadas nesta pesquisa: vulnerabilidade ampla, territorial e associada às condições de saúde. Para tal, são sugeridas pesquisas que se valham de dados primários para retratar com maior acurácia os aspectos levantados para o cálculo das vulnerabilidades socioeconômicas.

\section{REFERÊNCIAS}

ABREU, M. C. S.; ANDRADE, R. J C. Dealing with wicked problems in socio-ecological systems affected by industrial disasters: A framework for collaborative and adaptive governance. Science of the total environment, v. 694, p. 133700, 2019. https://doi.org/10.1016/i.scitotenv.2019.133700

ADHIKARI, R. P. et al. Health and Social Vulnerability of Adolescents in Nepal. SM J Public Health Epidemiol, v. 2, n. 3, p. 1032, 2016.

ASSOCIAÇÃO BRASILEIRA DE BARES E RESTAURANTES (ABRASEL). Carta aberta às instituições bancárias. 2020. Disponível em: https://abrasel.com.br/. Acessado em 27 mar. 2020.

ATLAS. Atlas do desenvolvimento humano no Brasil. 2013. Disponível em: <http://atlasbrasil.org.br/2013/>. Acessado em: 08 abr. 2020.

BAKER, J. L. (Ed.). Climate change, disaster risk, and the urban poor: cities building resilience for a changing world. New York: The World Bank, 2012. https://doi.org/10.1596/978-0-8213-8845-7

BRASIL, MINISTÉRIO DA SAÚDE (MS). Covid-19: Painel Coronavírus. Disponível em: <https://covid.saude.gov.br/>. Acessado em: 28 mar. 2020.

CÂMARA, S. F. et al. Vulnerabilidade socioeconômica à COVID-19 no epicentro da região Nordeste do Brasil (Ceará). Revista de Administração Pública, 2020. https://doi.org/10.1590/0034-761220200133

CROWLEY, K.; HEAD, B. W. The enduring challenge of 'wicked problems': revisiting Rittel and Webber. Policy Sciences, v. 50, n. 4, p. 539-547, 2017. https://doi.org/10.1007/s11077-017-9302-4

CUTTER, S. L. et al. A place-based model for understanding community resilience to natural disasters. Global environmental change, v. 18, n. 4, p. 598-606, 2008. https://doi.org/10.1016/i.gloenvcha.2008.07.013 
Samuel Façanha Câmara Hermano José Batista de Carvalho Felipe Gerhard

Vulnerabilidade socioeconômica da cidade de Fortaleza ao Felipe Roberto da Silva

DE LANGE, H. J. et al. Ecological vulnerability in risk assessment - a review and perspectives. Science of the total environment, v. 408, n. 18, p. $3871-3879, \quad 2010$. https://doi.org/10.1016/i.scitotenv.2009.11.009

EMRICH, C. T.; CUTTER, S. L. Social vulnerability to climate-sensitive hazards in the southern United States. Weather, Climate, and Society, v. 3, n. 3, p. 193-208, 2011. https://doi.org/10.1175/2011WCAS1092.1

HAND, M. S. et al. Socioeconomic vulnerability to ecological changes to National Forests and Grasslands in the Southwest. Gen. Tech. Rep. RMRS-GTR-383. Fort Collins, CO: US Department of Agriculture, Forest Service, Rocky Mountain Research Station. 100 p., v. 383, 2018. https://doi.org/10.2737/RMRS-GTR-383

HE, L.; SHEN, J.; ZHANG, Y. Ecological vulnerability assessment for ecological conservation and environmental management. Journal of environmental management, v. 206, p. 1115-1125, 2018. https://doi.org/10.1016/j.jenvman.2017.11.059

HEAD, B. W.; ALFORD, J. Wicked problems: Implications for public policy and management. Administration \& society, v. 47, n. 6, p. 711-739, 2015. https://doi.org/10.1177/0095399713481601

HOM, J. K. et al. Increased Health and Social Vulnerability Among Hepatitis C Infected Individuals Coinfected with Hepatitis B. Journal of health care for the poor and underserved, v. 29, n. 4, p. 12691280, 2018. https://doi.org/10.1353/hpu.2018.0094

INSTITUTO BRASILEIRO DE GEOGRAFIA E ESTATÍSTICA (IBGE). IBGE Cidades. 2020. Disponível em: <https://cidades.ibge.gov.br/>. Acessado em: 6 mar. 2020.

INSTITUTO BRASILEIRO DE GEOGRAFIA E ESTATÍSTICA (IBGE). Censo Demográfico 2010. Rio de Janeiro: IBGE, 2010. Disponível em: <http://censo2010.ibge.gov.br>. Acesso em: 31 mar. 2020.

INSTITUTO DE PESQUISA DO CEARÁ (IPECE). Informe 42: Perfil Municipal de Fortaleza. Tema VII: Distribuição Espacial da Renda Pessoal. Fortaleza, 2012.

INSTITUTO DE PLANEJAMENTO DE FORTALEZA (IPLANFOR). Plano Fortaleza 2040: equidade social, territorial e econômica. Prefeitura Municipal de Fortaleza: IPLANFOR, 2016.

INSTITUTO DE PLANEJAMENTO DE FORTALEZA (IPLANFOR). Relatório das ZEIS: comitê técnico intersetorial e comunitário da ZEIS. Fortaleza: IPLANFOR, 2015.

INTERNATIONAL LABOUR ORGANIZATION (ILO). COVID-19 and the world of work: Impact and policy $\quad 2020 . \quad$ responses. 2 isponível: $\quad$ https://www.ilo.org/global/about-theilo/newsroom/news/WCMS_738742/lang--en/index.htm>. Acessado em: 05 abr. 2020.

JIA, L. et al. Prediction and analysis of Coronavirus Disease 2019. Cornell University, arXiv preprint arXiv:2003.05447, 2020.

KRAEMER, M. U. et al. The effect of human mobility and control measures on the COVID-19 epidemic in China. Science, v. 368, n. 6490, p. 493-497, 2020. https://doi.org/10.1126/science.abb4218

KREUTER, M. W. et al. Understanding wicked problems: a key to advancing environmental health promotion. Health education \& behavior, v. 31, n. 4, p. 441-454, 2004. https://doi.org/10.1177/1090198104265597

LALL, S. V.; DEICHMANN, U. Density and disasters: economics of urban hazard risk. The World Bank, 2010. https://doi.org/10.1596/1813-9450-5161

$\mathrm{LI}$, R. et al. Substantial undocumented infection facilitates the rapid dissemination of novel coronavirus (SARS-CoV-2). Science, v. 368, n. 6490, p. 489-493, 2020. https://doi.org/10.1126/science.abb3221

LINS-DE-BARROS, F. M. Integrated coastal vulnerability assessment: A methodology for coastal cities management integrating socioeconomic, physical and environmental dimensions-Case study of Região dos Lagos, Rio de Janeiro, Brazil. Ocean \& coastal management, v. 149, p. 1-11, 2017. https://doi.org/10.1016/j.ocecoaman.2017.09.007

MADHAV, N. et al. Pandemics: risks, impacts, and mitigation. In: Jamison D. T. et al. (eds.). Disease Control Priorities: Improving Health and Reducing Poverty. 3rd edition. The International Bank for 
Samuel Façanha Câmara

Hermano José Batista de Carvalho Felipe Gerhard

Vulnerabilidade socioeconômica da cidade de Fortaleza ao Felipe Roberto da Silva

Reconstruction and Development/The World Bank, 2017. https://doi.org/10.1596/978-1-4648-05271 ch17

MALHOTRA, N. K. Pesquisa de marketing: foco na decisão. 3. ed. São Paulo: Pearson Prentice Hall, 2011.

MCKERCHER, B. SIP (Sars induced Panic) a greater threat to Tourism than Sars (Severe acute respiratory Syndrome. E-revise of Tourism Research (ERTR), v. 1, n. 1, p. 17-18, 2003.

MCLAFFERTY, S. Placing pandemics: geographical dimensions of vulnerability and spread. Eurasian geography and economics, v. 51, n. 2, p. 143-161, 2010. https://doi.org/10.2747/1539-7216.51.2.143

NEIDERUD, C. J. How urbanization affects the epidemiology of emerging infectious diseases. Infection ecology \& epidemiology, v. 5, n. 1, p. 27060, 2015. https://doi.org/10.3402/iee.v5.27060

NELSON, J. R.; GRUBESIC, T. H. Oil spill modeling: risk, spatial vulnerability, and impact assessment. Progress in Physical Geography: Earth and Environment, v. 42, n. 1, p. 112-127, 2018. https://doi.org/10.1177/0309133317744737

NELSON, J. R. et al. A geospatial evaluation of oil spill impact potential on coastal tourism in the Gulf of Mexico. Computers, Environment and Urban Systems, v. 68, p. 26-36, 2018. https://doi.org/10.1016/j.compenvurbsys.2017.10.001

NGUYEN, A. K. et al. A. Zoning eco-environmental vulnerability for environmental management and $\begin{array}{llllll}\text { protection. Ecological Indicators, } & \text { v. } & 69, & \text { p. } & 100-117, & \end{array}$ https://doi.org/10.1016/j.ecolind.2016.03.026

PATNAIK, U.; NARAYANAN, K. Vulnerability and climate change: An analysis of the eastern coastal districts of India. Disponível em: https://mpra.ub.uni-muenchen.de/22062/. Acesso em: 21 mar. 2020. 2009.

PAVEGLIO, T. B. et al. Evaluating the characteristics of social vulnerability to wildfire: demographics, perceptions, and parcel characteristics. Environmental management, v. 58, n. 3, p. 534-548, 2016. https://doi.org/10.1007/s00267-016-0719-x

PREFEITURA MUNICIPAL DE FORTALEZA (PMF). Índice de Desenvolvimento Humano (IDH). Fortaleza: Secretaria municipal de Desenvolvimento Econômico, 2014.

PREFEITURA MUNICIPAL DE FORTALEZA (PMF). Plano Fortaleza 2040. 2020. Disponível em: <https://fortaleza2040.fortaleza.ce.gov.br/site/>. Acessado em: abr. 2020.

REINICKE, W. H.; BENNER, T.; WITTE, J. M. Global public policy: Globalisierung gestalten durch globale politiknetzwerke. Networks, v. 117, p. 44-57, 2001.

RELAÇÃO ANUAL DE INFORMAÇÕES SOCIAIS (RAIS). Bases estatísticas RAIS/CAGED. Disponível em: <http://bi.mte.gov.br/bgcaged/login.php>. Acessado em: 20 mar. 2020.

RITTEL, H. W. J.; WEBBER, M. M. Dilemmas in a general theory of planning. Policy sciences, v. 4, n. 2, p. 155-169, 1973. https://doi.org/10.1007/BF01405730

SANDS, P. et al. Assessment of economic vulnerability to infectious disease crises. The Lancet, v. 388, n. 10058 , p. 2443-2448, 2016. https://doi.org/10.1016/S0140-6736(16)30594-3

SECRETARIA DA SAÚDE DO ESTADO DO CEARÁ (SESA). Arquivos Coronavírus (Covid-19). Boletim epidemiológico novo coronavírus (Covid-19) - 26 de março de 2020. 2020. Disponível em: <https://www.saude.ce.gov.br/download/arquivos-coronavirus-covid-19/>. Acessado em: 27 mar. 2020.

SECRETARIA DE DESENVOLVIMENTO ECONÔMICO DE FORTALEZA (SDE). Mapas, índices e indicadores de $\quad$ Fortaleza. 2019 . Disponível em: < https://public.tableau.com/profile/secretaria.de.desenvolvimento.economico.sde\#!/>. Acesso em: 22 mar. 2020.

SECRETARIA MUNICIPAL DE SAÚDE (SMS). 2020. Boletins epidemiológicos. Disponível em: $<$ https://coronavirus.fortaleza.ce.gov.br/boletim-epidemiologico.html>. Acessado em: 20 abr. 2020. 
SILVA, M. M. G. T.; KAWASAKI, A. Socioeconomic vulnerability to disaster risk: a case study of flood and drought impact in a rural Sri Lankan community. Ecological Economics, v. 152, p. 131-140, 2018. https://doi.org/10.1016/j.ecolecon.2018.05.010

SINGLETON, A. D. et al. Measuring the spatial vulnerability of retail centres to online consumption through a framework of e-resilience. Geoforum, v. 69, p. 5-18, 2016.

https://doi.org/10.1016/i.geoforum.2015.11.013

SIU, A.; WONG, Y. C. R. Economic impact of SARS: the case of Hong Kong. Asian Economic Papers, v. 3, n. 1, p. 62-83, 2004. https://doi.org/10.1162/1535351041747996

SUMMER, A.; HOY, C.; ORTIZ-JUAREZ, E. Estimates of the Impact of COVID-19 on Global Poverty. UNU-WIDER WIDER Working Paper, April, p. 1-14, 2020. https://doi.org/10.35188/UNUWIDER/2020/800-9

TIBÚRCIO, L. H.; CORRÊA, M. P. Análise da vulnerabilidade da microrregião de Itajubá por meio do IVG com vistas à mitigação dos impactos causados pelas mudanças climáticas. Ambiente \& Sociedade, v. 15, n. 3, p. 123-139, 2012. https://doi.org/10.1590/S1414-753X2012000300008

UNIVESIDADE DE JOHN HAPKINS (JHU). COVID-19 Dashboard by the Center for Systems Science and Engineering (CSSE). Disponível em: <https://www.arcgis.com/apps/opsdashboard/index.html\#/bda7594740fd40299423467b48e9ecf6 >. Acessado em: 29 mar. 2020.

WALTNER-TOEWS, D. Zoonoses, One Health and complexity: wicked problems and constructive conflict. Philosophical Transactions of the Royal Society B: Biological Sciences, v. 372, n. 1725, p. 20160171, 2017. https://doi.org/10.1098/rstb.2016.0171

WU, S. Y.; YARNAL, B.; FISHER, A. Vulnerability of coastal communities to sea-level rise: a case study of Cape May County, New Jersey, USA. Climate Research, v. 22, n. 3, p. 255-270, 2002. https://doi.org/10.3354/cr022255

XIANG, W. N. Working with wicked problems in socio-ecological systems: Awareness, acceptance, and adaptation. Landscape and Urban Planning, n. 110, p. 1-4, 2013. https://doi.org/10.1016/i.landurbplan.2012.11.006

Recebido em: 13/05/2020

Aceito para publicação em: 21/08/2020 